Cite as: F. Huber et al., Science

10.1126/science.aay3444 (2019).

\title{
Chemical bond formation showing a transition from physisorption to chemisorption
}

\author{
Ferdinand Huber' ${ }^{1}$, Julian Berwanger ${ }^{1}$, Svitlana Polesya ${ }^{2}$, Sergiy Mankovsky² ${ }^{2}$, Hubert Ebert ${ }^{2}$, Franz J. Giessibl ${ }^{1 *}$ \\ ${ }^{1}$ Institute of Experimental and Applied Physics, Department of Physics, University of Regensburg, 93040 Regensburg, Germany. ${ }^{2}$ Department of Chemistry, \\ Ludwig-Maximilians-Universität München, 81377 Munich, Germany. \\ *Corresponding author. Email: franz.giessibl@ur.de
}

Surface molecules can transition from physisorption through weak van-der-Waals forces to a strongly bound chemisorption state by overcoming an energy barrier. We show that a $\mathrm{CO}$ molecule adsorbed to the tip of an atomic force microscope enables a controlled observation of bond formation, including its potential transition from physisorption to chemisorption. During imaging of $\mathrm{Cu}$ and $\mathrm{Fe}$ adatoms on a $\mathrm{Cu}(111)$ surface, the $\mathrm{CO}$ was not chemically inert but transited through a physisorbed local energy minimum into a chemisorbed global minimum, and an energy barrier was seen for the Fe adatom. Density functional theory reveals that the transition occurs through a hybridization of the electronic states of the $\mathrm{CO}$ molecule mainly with $s^{-}, p_{z^{-}}$and $d_{z}^{2}$-type states of the Fe and $\mathrm{Cu}$ adatoms, leading to chemical bonding.

The physicist Richard Feynman believed that the sentence "...all things are made of atoms-little particles that move around in perpetual motion, attracting each other when they are a little distance apart, but repelling upon being squeezed into one another" (1) contains the most information about scientific knowledge in the fewest words. While this quote captures the key characteristics of chemical bonding, subtle complications do occur in nature. Lennard-Jones already described in 1932 [see figure 3 in (2)] that molecules can bond in two ways to a surface, a weak type induced by van-derWaals (vdW) attraction (physisorption) and, for smaller distances, a stronger chemical bond (chemisorption). In some cases, these two bonding regimes are split by an energetic barrier and depending on the height of the barrier, transitions can occur [see figure 9.5 in (3)]. Overall, three different bonding scenarios can evolve $(3,4)$ :

1) The formation of a weak physical bond (vdW bond) with depth of about $20 \mathrm{meV}(0.46 \mathrm{kcal} / \mathrm{mol})$ as shown by the potential energy $V$ versus distance $z$ curve in Fig. 1A and its corresponding force curve $F_{z}(z)$ in Fig. 1D with a maximal attractive force (5) on the order of $10 \mathrm{pN}$. The interaction of two noble gas atoms such as Xe is an example for such an interaction.

2) The formation of a strong chemical bond with energies on the order of electron volts shown in Fig. 1B, where the attractive force (Fig. 1E) can reach nanonewtons and mask the ever present vdW forces that are on the order of $10 \mathrm{pN}$, followed by repulsion at small z. The data in Fig. 1, B and E, correspond to the bonding energy and vertical force between two Si atoms according to the Stillinger-Weber potential (6).

3) The third bonding mechanism involves a transition from physisorption to chemisorption as shown in Fig. 1C (3,
4). The initial appearance of a weak vdW bond is followed by a transition that can show an energy barrier of high strength (black curve of Fig. 1C), medium (green and red) and vanishing barrier (blue). If a molecule arrives at the surface with sufficient thermal energy to overcome the slight energy barrier of the green energy curve in Fig. 1C, it can chemisorb immediately. If a stronger energy barrier occurs as shown by the black line of Fig. 1C, its energy needs to be lifted by thermal excitation to overcome the barrier and to form a strong chemical bond (Fig. 1F). The $V(z)$ curve in Fig. $1 \mathrm{C}$ is key to the physisorption-chemisorption transition and possible subsequent heterogeneous catalysis. While previous methods only provided the equilibrium positions at their corresponding temperatures, state-of-the-art atomic force microscopy at low temperatures can directly record this curve.

Carbon monoxide (CO) can undergo physisorption as well as molecular and dissociative chemisorption on transition metal surfaces. Dissociative chemisorption to adsorbed $\mathrm{C}$ and $\mathrm{O}$ atoms tends to prevail on all transition metals in the periodic table left of a boundary between iron and cobalt at room temperature [chapter 6.4 .1 in $(4)$ and $(7,8)$ ], as well as for W (9). Conventional methods for adsorption studies such as thermal desorption spectroscopy or electron energy loss spectroscopy $(3,4)$ probe large molecular ensembles. Chemisorption is key to heterogeneous catalysis and detailed knowledge about its basic mechanism can be obtained by using scanning tunneling microscopy (STM) as an atomic probe (10). Although STM combined with ultrashort laser pulsing has recently obtained femtosecond time resolution in imaging the surface vibrations of molecules (11), so far STM has been used to image the end products of surface reactions and not the reactions itself. 
Atomic force microscopy (AFM) (12) and its variants (13, 14) have become a powerful tool for surface studies (15). The attachment of a CO molecule to an STM tip can enhance resolution by in effect creating a sharper probe tip (16), and Gross et al. reported that CO-terminated AFM tips allow imaging of organic molecules with intramolecular resolution (17), leading to a wide use of CO-terminated tips (18). The inertness of CO-terminated tips enabled imaging of many organic molecules (18), graphene (19) as well as metal clusters and the silicon $(111)-(7 \times 7)$ surface $(20)$ at unprecedented resolution. The use of CO-terminated AFM tips allows the tracking of the formation and potential transition from physisorption to chemisorption of a bond as a function of distance (i.e., reaction coordinate) for a single $\mathrm{CO}$ molecule with a precisely controlled position on a picometer scale.

There is a restriction imposed by the bond of the $\mathrm{CO}$ molecule to the tip. A CO molecule in the gas phase can orient itself freely on a surface to enable the maximal bonding strength. In metal carbonyls such as $\mathrm{Ni}(\mathrm{CO})_{4}$ or $\mathrm{Fe}(\mathrm{CO})_{5}$, $\mathrm{CO}$ bonds with the $\mathrm{C}$ atom to the transition metal (21) and $\mathrm{CO}$ bonds to the AFM's metal tip in a similar manner. Experimental and theoretical evidence holds that the oxygen end of the CO-terminated tip is chemically inert. When imaging pentacene with CO-terminated tips (17), density functional theory (DFT) has shown that Pauli repulsion between electrons provides the contrast $(22,23)$.

The bottom row in Fig. 1 displays experimental $F_{z}(z)$ curves over the centers of three different adatoms obtained with CO-terminated tips. Figure $1 \mathrm{G}$ shows the interaction of a CO-terminated tip with a single Si adatom on $\mathrm{Cu}(111)$ as indicated in the inset. The attractive vdW force reached only 20 pN before Pauli repulsion forces dominate (24). The interaction of the CO-terminated tip with the Si adatom resembled physisorption-a weak attraction turns to Pauli repulsion with a single energetic minimum. The strong covalent bonds shown in Fig. 1, B and E, with a magnitude of nanonewtons were used to atomically resolve AFM images in vacuum on the silicon surface (25), where DFT identified a covalent character (26) that was verified by precise force spectroscopy ( 27 , 28).

Figure $1 \mathrm{H}$ shows the $F_{z}(z)$ curve for a CO-terminated tip over a $\mathrm{Cu}$ adatom on $\mathrm{Cu}(111)$. The attractive force minimum was at $z=373 \mathrm{pm}$, and the attractive $z$-range was widened compared to the Si curve in Fig. 1G. Figure 1I shows the $F_{z}(z)$ curve for a CO-terminated tip over a Fe adatom on $\mathrm{Cu}(111)$, which resembles the qualitative physisorption-chemisorption transition of Fig. $1 \mathrm{~F}$ (black curve). The physisorbed force minimum of $-8 \mathrm{pN}$ at $z=420 \mathrm{pm}$ was followed by a force barrier of $+17 \mathrm{pN}$ at $z=310 \mathrm{pm}$ and a maximal attractive force of $-364 \mathrm{pN}$ at $z=250 \mathrm{pm}$. The occurrence of a barrier in the experimental force curves for the Fe adatom in Fig. 1I and its similarity to the schematic force curves pertaining to a physisorption-chemisorption transition in Fig. $1 \mathrm{~F}$ points to the experimental observation of such a transition, as elucidated below.

The $F_{z}(z)$ curves in Fig. 1, G to I, were measured with the CO-terminated tip exactly centered over the adatoms. However, $F$ is not merely a function of absolute distance between the centers of the $\mathrm{O}$ atom of the tip and the adatom, but also of the polar and azimuthal angles with respect to the surface normal and substrate orientation. The top row of Fig. 2 shows $F$ in the $z$-direction as a function of lateral $x$-direction and $z$ position at $y=0$. The force fields for the three different adatoms were distinctly different in the $x z$-plane. The force curves in Fig. 1, G to I, are traces of the two-dimensional force fields $F_{z}(x, y, z)$ at $x=y=0$. The central row shows experimental constant-height force images of the three adatoms. The bottom row displays DFT force calculations for the three different adatoms.

The left column in Fig. 2 shows data for the simplest case, the $\mathrm{Si}$ adatom. For the force data of the $\mathrm{Si}$ adatom on the $x z$ plane in Fig. 2A, we initially found weak vdW attraction followed by strong Pauli repulsion that was roughly proportional to the total charge density of the $\mathrm{Si}$ adatom as displayed in fig. S1A. The Si adatom appeared in the $x y$-plane (Fig. 2B) as a Gaussian-shaped repulsion, showing that the CO-terminated tip interacted with it in a similar manner as with organic molecules.

Simulations of this image for four different heights using the so-called Probe Particle Model $(29,30)$ are shown in fig. S2 where the lateral bending of the CO-terminated tip (31) was taken into account. The DFT calculation of the force image (Fig. 2C) yielded a similar result as the experimental data (32). Because Pauli repulsion was the contrast mechanism here, the experimental images resembled the total charge densities presented in fig. S1A. DFT confirmed that Pauli repulsion was the contrast mechanism-differential charge density plots and calculations of the energies of the states (see figs. S7, A to D, and S8, A to F) showed no evidence for chemical bonding.

For the $\mathrm{Cu}$ adatom data (central column of Fig. 2), in the center at $x \approx 0$ in Fig. 2D, vdW attraction was followed by some more slight attraction before turning to Pauli repulsion. The circumference of the $\mathrm{Cu}$ adatom at $x \approx \pm 200$ pm looked completely different with a transition from vdW attraction directly to Pauli repulsion. Accordingly, the constant height data in Fig. 2E showed a ring-like appearance. The DFT calculation in Fig. $2 \mathrm{~F}$ resembled the experimental data in Fig. $2 \mathrm{E}$ and was vastly different from the total charge density of the $\mathrm{Cu}$ adatom shown in fig. S1B. The evolution of the contrast with distance starts from the attractive vdW signature, changes to the repulsive ring, and ends in a repulsive cusp in the center as shown in detail in fig. S3. The calculated $F_{z}(z)$ curves (fig. S7E), differential charge density plots (fig. S7, F to 
$\mathrm{H})$ and pronounced shifts in the energies of the electronic states (fig. S8, G to M) provided a consistent data set indicating the emergence of a bond with medium strength (33). The physical origin for the delayed transition from vdW attraction to Pauli repulsion is a hybridization of the electronic states of the $\mathrm{Cu}$ adatom with the states of the CO-terminated tip (34).

For the Fe adatom (right column of Fig. 2), in the center at $x \approx 0$, the interaction started with vdW attraction (dark lentil shaped area at $z \approx 400 \mathrm{pm}$ ), followed by weak repulsion (light green lentil shaped area at $z \approx 330 \mathrm{pm}$ ). After penetrating the repulsive barrier in the center, attraction occurred (see also Fig. 1I). For even smaller $z$, we expected repulsion again but this close distance is not accessible because approaching to such small distances risked the integrity of the CO-terminated tip (35). Outside the center, at $x \approx \pm 210 \mathrm{pm}$, we saw a direct transition from vdW attraction to Pauli repulsion similar to the circumference of the $\mathrm{Cu}$ adatom. The top view at Fig. $2 \mathrm{H}$ showed a repulsive ring similar to the $\mathrm{Cu}$ adatom, but for the Fe adatom, three local maxima were located over the hollow sites of the underlying $\mathrm{Cu}(111)$ surface (see figs. S5 and S6). The DFT force calculations presented in Fig. 2I confirmed the presence of three local maxima on the repulsive ring in registry with the $\mathrm{Cu}(111)$ substrate (see fig. S6).

As in the case of the $\mathrm{Cu}$ adatom, the images of the Fe adatom did not relate to the total charge density of the Fe adatom shown in fig. S1C. The physical origin of the ring-like appearance and strong attraction in the center of the Fe adatom was a hybridization of electronic states between tip and sample as revealed by the DFT calculations. The $F_{z}(z)$ curves (fig. S7I), differential charge density plots (fig. S7, J to L), and pronounced shifts in the energies of the electronic states of the CO-terminated tip and Fe adatom (fig. S8, $\mathrm{N}$ to $\mathrm{T}$ ) provided a coherent picture of the formation of a chemical bond due to hybridization (33). We note that the appearance of the $\mathrm{Cu}$ and $\mathrm{Fe}$ adatoms as repulsive tori are not an artifact of bending of the CO-terminated tip (31) (fig. S9).

The experimental images of $\mathrm{Cu}$ and $\mathrm{Fe}$ adatoms showed similarities and differences. Both appear as repulsive tori when imaged with CO-terminated tips at close distance. However, the Fe adatom showed three distinctive local maxima on the torus, and the attractive force in the center reached values down to $-364 \mathrm{pN}$, while the center of the $\mathrm{Cu}$ adatom was much less attractive and even allowed to image the repulsive cusp for very small distances. Previous experiments have shown that single $\mathrm{Fe}$ adatoms on $\mathrm{Cu}(111)$ have a magnetic moment (36). Our DFT calculations confirm this and find zero magnetic moment for the $\mathrm{Cu}$ adatom. Thus, the physical origin of the difference in the AFM data of $\mathrm{Cu}$ versus Fe adatoms is the element-specific occupation of the majority and minority $3 d$ spin states (see fig. S12).
We have shown that CO-terminated tips can hybridize with sample atoms and produce a contrast much different from the total charge density. The subatomic contrast (20), i.e., the appearance of nontrivial structures within images of single atoms, was explained as a signature of hybridization of states with a $s, p$ and $d$ character in the formation of chemical bonds. The present findings extend atomically resolved force microscopy into a new interaction regime. When atomically resolved AFM in vacuum was introduced 25 years ago, strong covalent or ionic bonds were probed in a noncontact distance regime, and noncontact AFM and atomically resolved AFM have historically often been used as synonyms. The introduction of CO-terminated tips by Gross et al. (17) as well as noble gas and other inert tips (37) expanded the distance regime where nondestructive atomically resolved images is possible from the noncontact regime to an intermittent-contact mode that probes Pauli repulsion forces.

The present work further expands AFM into a distance regime where the hybridizations occur that underlie the chemical bond. Possible applications include the study of partially unfilled $\mathrm{Cu} 3 d$ states in cuprate superconductors (38). Finally, we showed that CO-terminated tips are not generally chemically inert as tips terminated by noble gas atoms. Therefore, CO-terminated tips do not generally interact via Pauli repulsion with the total charge density of the sample. This might change the interpretation of images of organic molecules that contain metal ions, in particular those with unfilled $3 d$ shells.

\section{REFERENCES AND NOTES}

1. R. P. Feynman, R. B. Leighton, M. Sands, The Feynman Lectures on Physics I (Addison-Wesley, 1963), chaps. 1-2.

2. J. E. Lennard-Jones, Processes of adsorption and diffusion on solid surfaces. Trans. Faraday Soc. 28, 333-359 (1932). doi:10.1039/tf9322800333

3. A. Zangwill, Physics at Surfaces (Cambridge Univ. Press, 1988).

4. H. Ibach, Physics of Surfaces and Interfaces (Springer, 2006).

5. The potential energy $V$ of a bond as a function of distance $z$ between the atoms has its minimum at the bonding distance $z=\sigma$. Here, we display the force $F_{z}$ that is given by the negative derivative of the potential energy with respect to distance with $F_{z}=-\partial V / \partial z$ with $F_{z}(\sigma)=0$. The shapes of the $F_{z}(z)$ and $V(z)$ curves are very similar except for a lateral shift-while $F_{z}(\sigma)=0, V(\sigma)$ is the minimal energy. This similarity of overall shape and lateral shift also holds for more complex potentials that involve a repulsive barrier.

6. F. H. Stillinger, T. A. Weber, Computer simulation of local order in condensed phases of silicon. Phys. Rev. B Condens. Matter 31, 5262-5271 (1985). doi:10.1103/PhysRevB.31.5262 Medline

7. G. Brodén, T. N. Rhodin, C. Brucker, R. Benbow, Z. Hurych, Synchrotron radiation study of chemisorptive bonding of $\mathrm{CO}$ on transition metals-Polarization effect on Ir(100). Surf. Sci. 59, 593-611 (1976). doi:10.1016/0039-6028(76)90038-8

8. S.-S. Sung, R. Hoffmann, How carbon monoxide bonds to metal surfaces. J. Am. Chem. Soc. 107, 578-584 (1985). doi:10.1021/ja00289a009

9. F. M. Propst, T. C. Piper, Detection of the vibrational states of gases adsorbed on tungsten by low energy electron scattering. J. Vac. Sci. Technol. 4, 53-56 (1967). doi:10.1116/1.1492522

10. G. Ertl, Reactions at surfaces: From atoms to complexity (Nobel Lecture). Angew. Chem. Int. Ed. 47, 3524-3535 (2008). doi:10.1002/anie.200800480 Medline 
11. T. L. Cocker, D. Peller, P. Yu, J. Repp, R. Huber, Tracking the ultrafast motion of a single molecule by femtosecond orbital imaging. Nature 539, 263-267 (2016). doi:10.1038/nature19816 Medline

12. G. Binnig, C. F. Quate, C. Gerber, Atomic force microscope. Phys. Rev. Lett. 56, 930-933 (1986). doi:10.1103/PhysRevLett.56.930 Medline

13. T. R. Albrecht, P. Grütter, D. Horne, D. Rugar, Frequency modulation detection using high-Q cantilevers for enhanced force microscope sensitivity. J. Appl. Phys. 69, 668-673 (1991). doi:10.1063/1.347347

14. U. Dürig, O. Züger, A. Stalder, Interaction force detection in scanning probe microscopy: Methods and applications. J. Appl. Phys. 72, 1778-1798 (1992). doi:10.1063/1.352348

15. R. García, R. Pérez, Dynamic atomic force microscopy methods. Surf. Sci. Rep. 47 , 197-301 (2002). doi:10.1016/S0167-5729(02)00077-8

16. L. Bartels, G. Meyer, K.-H. Rieder, Controlled vertical manipulation of single CO molecules with the scanning tunneling microscope: A route to chemical contrast. Appl. Phys. Lett. 71, 213-215 (1997). doi:10.1063/1.119503

17. L. Gross, F. Mohn, N. Moll, P. Liljeroth, G. Meyer, The chemical structure of a molecule resolved by atomic force microscopy. Science 325, 1110-1114 (2009). doi:10.1126/science.1176210 Medline

18. N. Pavliček, L. Gross, Generation, manipulation and characterization of molecules by atomic force microscopy. Nat. Rev. Chem. 1, 0005 (2017). doi:10.1038/s41570-016-0005

19. M. P. Boneschanscher, J. van der Lit, Z. Sun, I. Swart, P. Liljeroth, D. Vanmaekelbergh, Quantitative atomic resolution force imaging on epitaxial graphene with reactive and nonreactive AFM probes. ACS Nano 6, 10216-10221 (2012). doi:10.1021/nn3040155 Medline

20. M. Emmrich, F. Huber, F. Pielmeier, J. Welker, T. Hofmann, M. Schneiderbauer, D. Meuer, S. Polesya, S. Mankovsky, D. Ködderitzsch, H. Ebert, F. J. Giessibl, Subatomic resolution force microscopy reveals internal structure and adsorption sites of small iron clusters. Science 348, 308-311 (2015). doi:10.1126/science.aaa5329 Medline

21. L. Pauling, The Nature of the Chemical Bond (Cornell Univ. Press, ed. 3, 1960).

22. N. Moll, L. Gross, F. Mohn, A. Curioni, G. Meyer, The mechanisms underlying the enhanced resolution of atomic force microscopy with functionalized tips. New J. Phys. 12, 125020 (2010). doi:10.1088/1367-2630/12/12/125020

23. N. Moll, L. Gross, F. Mohn, A. Curioni, G. Meyer, A simple model of molecular imaging with noncontact atomic force microscopy. New J. Phys. 14, 083023 (2012). doi:10.1088/1367-2630/14/8/083023

24. $\mathrm{CO}$ bending (31) is responsible for the experimental observation of a decreasing slope of the force curve in Fig. $1 \mathrm{G}$ for close distances.

25. F. J. Giessibl, Atomic resolution of the silicon (111)-(7×7) surface by atomic force microscopy. Science 267, 68-71 (1995). doi:10.1126/science.267.5194.68 Medline

26. R. Pérez, M. C. Payne, I. Štich, K. Terakura, Role of covalent tip-surface interactions in noncontact atomic force microscopy on reactive surfaces. Phys. Rev. Lett. 78, 678-681 (1997). doi:10.1103/PhysRevLett.78.678

27. M. A. Lantz, H. J. Hug, R. Hoffmann, P. J. A. van Schendel, P. Kappenberger, S. Martin, A. Baratoff, H.-J. Güntherodt, Quantitative measurement of short-range chemical bonding forces. Science 291, 2580-2583 (2001). doi:10.1126/science. 1057824 Medline

28. Y. Sugimoto, P. Pou, M. Abe, P. Jelinek, R. Pérez, S. Morita, O. Custance, Chemical identification of individual surface atoms by atomic force microscopy. Nature 446, 64-67 (2007). doi:10.1038/nature05530 Medline

29. P. Hapala, G. Kichin, C. Wagner, F. S. Tautz, R. Temirov, P. Jelínek, Mechanism of high-resolution STM/AFM imaging with functionalized tips. Phys. Rev. B 90, 085421 (2014). doi:10.1103/PhysRevB.90.085421

30. P. Hapala, R. Temirov, F. S. Tautz, P. Jelínek, Origin of high-resolution IETS-STM images of organic molecules with functionalized tips. Phys. Rev. Lett. 113, 226101 (2014). doi:10.1103/PhysRevLett.113.226101 Medline

31. L. Gross, F. Mohn, N. Moll, B. Schuler, A. Criado, E. Guitián, D. Peña, A. Gourdon, G. Meyer, Bond-order discrimination by atomic force microscopy. Science 337, 1326-1329 (2012). doi:10.1126/science.1225621 Medline

32. The apparent 6-fold symmetry in the DFT data is an artifact due to the limited number of data points.
33. R. Hoffmann, A chemical and theoretical way to look at bonding on surfaces. Rev. Mod. Phys. 60, 601-628 (1988). doi:10.1103/RevModPhys.60.601

34. In a previous publication (20), we provided a hypothesis to explain the ring-like structure of $\mathrm{Cu}$ and $\mathrm{Fe}$ adatoms that was compatible with the interpretation of $\mathrm{CO}$ terminated tips imaging the total charge density of the sample, similar to what was found by Moll et al. $(22,23)$ for organic molecules. The ring-like structure of $\mathrm{Fe}$ and $\mathrm{Cu}$ adatoms was explained by a sp-hybridization of the adatom's $4 s$ electrons to a $4 s p_{z}$ orbital that displays a torus-shaped total charge density when observed from above. However, the DFT calculations shown in figs. S1, S7, and S8 as well as the experimental observation of the repulsive barrier above the $\mathrm{Fe}$ adatom showed that the hybridization occurs only under the presence of the $\mathrm{CO}$ terminated tip and it not only involves $s$ and $p$ states, but also $d$ states.

35. The CO-terminated tip can come quite close to the Fe adatom when located at the center of the Fe adatom where lateral forces are zero. In Fig. 11, the tip was even approached almost to the equilibrium distance where the force is zero again after passing the maximal attraction of $-364 \mathrm{pN}$ at the distance of $250 \mathrm{pm}$. The minimal distance that can be sustained by the AFM tip without losing its $\mathrm{CO}$-termination is determined by experience. Usually, tip loss is immanent when the driving signal that controls the constant oscillation amplitude of the force sensor starts to rise, i.e., when damping of the sensor due to the tip-sample interaction becomes noticeable. When scanning in the $x y$-plane, lateral forces act on the $\mathrm{CO}$-terminated tip and larger distances are required to prevent the loss of the CO-termination (compare Fig. 2G where the minimal distance was almost 100 pm larger than in the force spectrum of Fig. 11).

36. G. E. Pacchioni, L. Gragnaniello, F. Donati, M. Pivetta, G. Autès, O. V. Yazyev, S. Rusponi, H. Brune, Multiplet features and magnetic properties of $\mathrm{Fe}$ on $\mathrm{Cu}(111)$ : From single atoms to small clusters. Phys. Rev. B 91, 235426 (2015). doi:10.1103/PhysRevB.91.235426

37. F. Mohn, B. Schuler, L. Gross, G. Meyer, Different tips for high-resolution atomic force microscopy and scanning tunneling microscopy of single molecules. Appl. Phys. Lett. 102, 073109 (2013). doi:10.1063/1.4793200

38. J. G. Bednorz, K. A. Müller, Perovskite-type oxides-the new approach to high- $T_{\mathrm{c}}$ superconductivity. Nobel Lecture. Angew. Chem. Int. Ed. 27, 735-748 (1988). doi:10.1002/anie.198807351

39. F. J. Giessibl, The qPlus sensor, a powerful core for the atomic force microscope. Rev. Sci. Instrum. 90, 011101 (2019). doi:10.1063/1.5052264 Medline

40. J. Welker, F. J. Giessibl, Revealing the angular symmetry of chemical bonds by atomic force microscopy. Science 336, 444-449 (2012). doi:10.1126/science. 1219850 Medline

41. J. E. Sader, S. Jarvis, Accurate formulas for interaction force and energy in frequency modulation force spectroscopy. Appl. Phys. Lett. 84, 1801-1803 (2004). doi:10.1063/1.1667267

42. J. E. Sader, B. D. Hughes, F. Huber, F. J. Giessibl, Interatomic force laws that evade dynamic measurement. Nat. Nanotechnol. 13, 1088-1091 (2018). doi:10.1038/s41565-018-0277-x Medline

43. I. Horcas, R. Fernández, J. M. Gómez-Rodríguez, J. Colchero, J. Gómez-Herrero, A. M. Baro, WSXM: A software for scanning probe microscopy and a tool for nanotechnology. Rev. Sci. Instrum. 78, 013705 (2007). doi:10.1063/1.2432410 Medline

44. M. Schneiderbauer, M. Emmrich, A. J. Weymouth, F. J. Giessibl, CO tip functionalization inverts atomic force microscopy contrast via short-range electrostatic forces. Phys. Rev. Lett. 112, 166102 (2014). doi:10.1103/PhysRevLett.112.166102 Medline

45. G. Kresse, J. Hafner, Ab initio molecular dynamics for liquid metals. Phys. Rev. $B$ Condens. Matter 47, 558-561 (1993). doi:10.1103/PhysRevB.47.558 Medline

46. G. Kresse, J. Hafner, Norm-conserving and ultrasoft pseudopotentials for first-row and transition elements. J. Phys. Condens. Matter 6, 8245-8257 (1994). doi:10.1088/0953-8984/6/40/015

47. J. P. Perdew, K. Burke, M. Ernzerhof, Generalized gradient approximation made simple. Phys. Rev. Lett. 77, 3865-3868(1996). doi:10.1103/PhysRevLett.77.3865 Medline

48. S. Grimme, J. Antony, S. Ehrlich, H. Krieg, A consistent and accurate ab initio parametrization of density functional dispersion correction (DFT-D) for the 94 elements H-Pu. J. Chem. Phys. 132, 154104 (2010). doi:10.1063/1.3382344 Medline 
49. A. J. Weymouth, T. Hofmann, F. J. Giessibl, Quantifying molecular stiffness and interaction with lateral force microscopy. Science 343, 1120-1122 (2014). doi:10.1126/science.1249502 Medline

50. M. Gajdoš, J. Hafner, $\mathrm{CO}$ adsorption on $\mathrm{Cu}(111)$ and $\mathrm{Cu}(001)$ surfaces: Improving site preference in DFT calculations. Surf. Sci. 590, 117-126 (2005). doi:10.1016/j.susc.2005.04.047

\section{ACKNOWLEDGMENTS}

We thank J. Repp and A. J. Weymouth for discussions, G. Ertl and R. Hoffmann for helpful comments, and F. Stilp for support in data acquisition. Funding: We thank the Deutsche Forschungsgemeinschaft for funding within the research Project No. CRC1277, subproject A02. Author contributions: F.H. recorded parts of the experimental data, performed most of the data analysis and visualized most of the data. J.B. recorded parts of the experimental data and validated the experimental data. S.P. and S.M. conducted all DFT calculations, analyzed the DFT results and visualized them. H.E. and F.J.G. are responsible for conceptualization, supervision of the project and for funding acquisition. F.J.G. prepared a figure and wrote the manuscript (original draft). All authors supported writing the manuscript (review and editing). Competing interests: All authors declare to have no competing interests, F.J.G. declares to hold patents for the force sensor that was used in the experiment. Data and materials availability: All relevant data are available in the main text or the supplementary materials. All raw data and scripts which were used for the data analysis are stored in the computer center of the University of Regensburg and are available on request.

\section{SUPPLEMENTARY MATERIALS}

science.sciencemag.org/cgi/content/full/science.aay3444/DC1

Materials and Methods

Figs. S1 to S12

References (39-50)

10 June 2019; accepted 29 August 2019

Published online 12 September 2019

10.1126/science.aay3444 

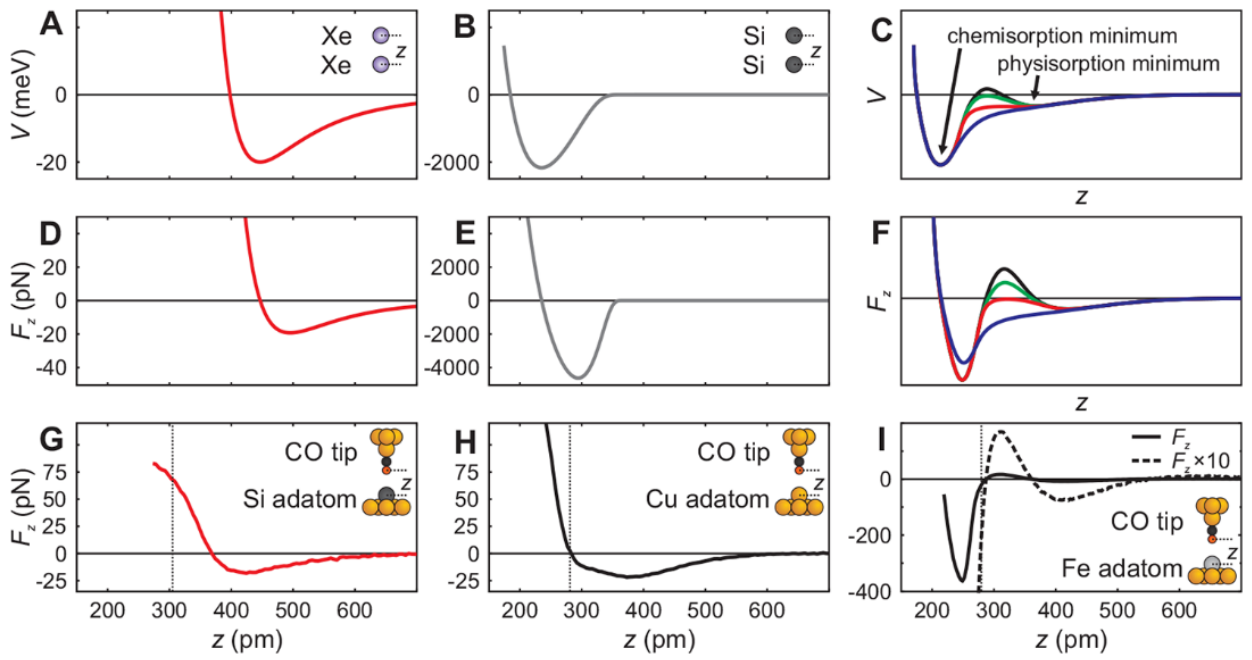

Fig. 1. Force and energy versus distance curves for various bonding situations. (A to $C$ ) Schematic potential energy $V$ and ( $D$ to $F$ ) vertical force $F_{z}$ versus distance $z$ curves for $(A$ and $D)$ a strong chemical bond, ( $B$ and $E$ ) a weak physical bond and ( $C$ and $F$ ) a bond with a transition from physisorption to chemisorption. The colored lines in (C) and (F) show four different cases that vary by their barrier height. If a repulsive energy barrier exists (i.e., $V>0$ in the region between physisorption and chemisorption as shown with a black line), the adsorbate may just reach the physisorbed state. For a very low energy barrier (green line), thermal excitation can suffice to bring the adsorbate in a chemisorbed state and for the red and blue lines, the adsorbate will immediately end up in the chemisorbed state. ( $G$ to I) Experimental force versus distance curves showing various bonds between the CO-terminated tip and a (G) Si adatom (24), (H) Cu adatom and (I) Fe adatom on $\mathrm{Cu}(111)$. The potential energy curve corresponding to (I) is shown in fig. S10. 

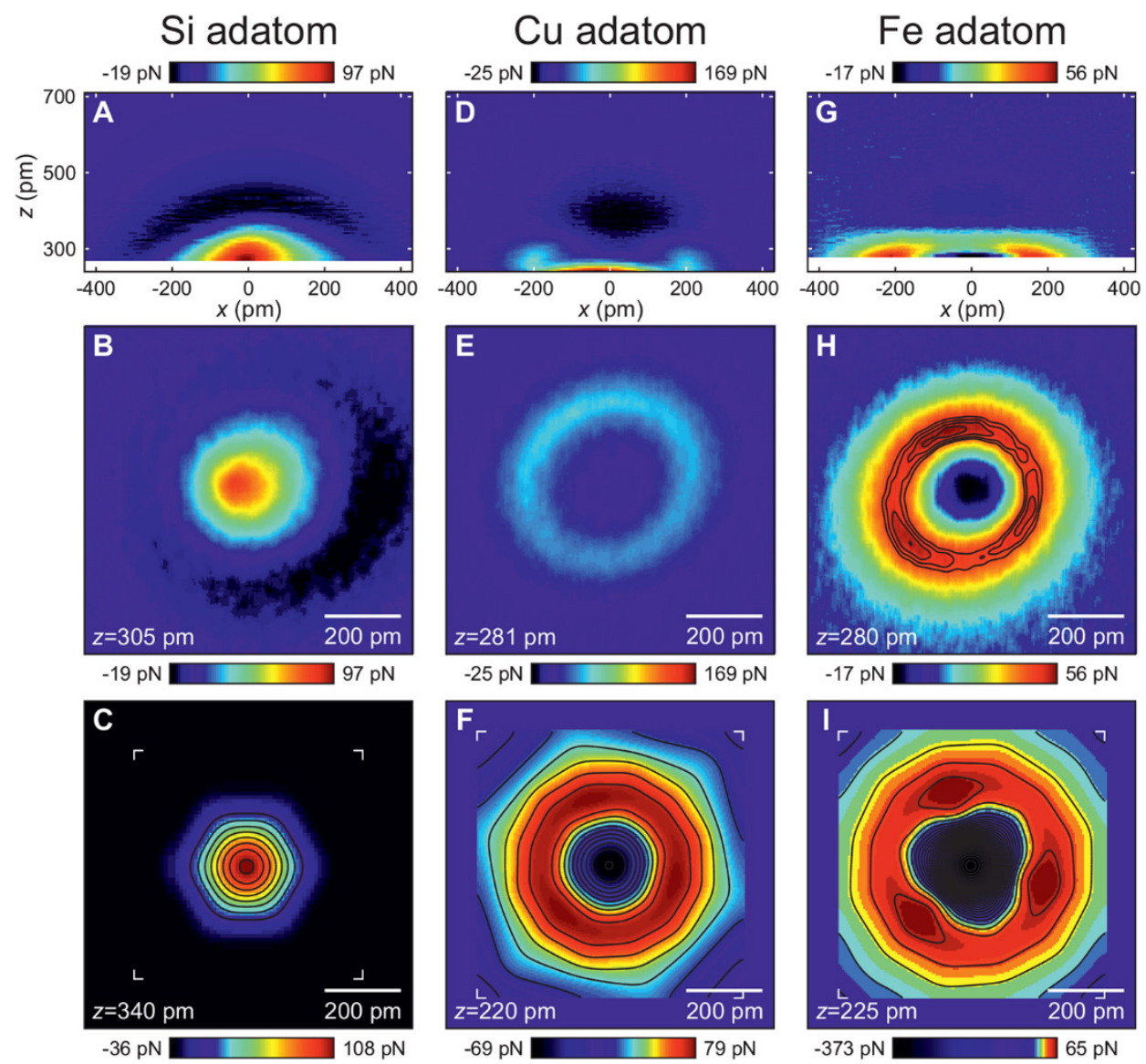

Fig. 2. Experimental and calculated forces for three different adatoms in side- and top view. Top row: Experimental vertical forces $F_{z}$ in the $x z$ plane between a CO-terminated AFM tip and a (A) Si adatom, (D) Cu adatom and $(\mathrm{G}) \mathrm{Fe}$ adatom on $\mathrm{Cu}(111)$. Middle row: Constant height force data in the $x y$-plane between a CO-terminated tip and (B) Si adatom, (E) Cu adatom and $(\mathrm{H}) \mathrm{Fe}$ adatom on $\mathrm{Cu}(111)$ taken at $z$-positions as indicated by vertical dotted lines in Fig. 1, G to I, respectively. Bottom row: Density functional theory calculations of $F_{z}$ in the $x y$-plane between a $\mathrm{CO}$ molecule tip and a (C) Si adatom, (F) Cu adatom and (I) Fe adatom on Cu(111). The three local maxima on the experimental $(\mathrm{H})$ and DFT data $(\mathrm{I})$ for the Fe adatom are located above the hollow sites of the $\mathrm{Cu}(111)$ substrate underneath (see figs. S5 and S6). Note that the color scale is the same for the force data in the top and center rows. The color scale in the bottom row is different in order to maximize the contrast. 